\title{
COVID-19, septiembre y nuestros niños, ¿qué hacer?, ¿cómo actuar?
}

\section{COVID-19, September and our children, what do do? how to act?}

\author{
PONTE-SUCRE A. ${ }^{1}$
}

\section{Resumen}

La temática del artículo está centrada en el compromiso que como sociedad tenemos con los niños y el futuro que a ellos pertenece. Se discute la situación de la pandemia de COVID-19 en el contexto de los niños y los adolescentes, y la necesidad de un cambio de paradigma mundial para preservar el planeta y su diversidad para ellos, con énfasis en el tema educativo y en la protección de la psicología emocional de los niños. Se incluye un llamado a entrenarnos en resiliencia.

Palabras clave: objetivos de sustentabilidad, pandemia, futuro de los niños, acciones a tomar

\begin{abstract}
The article focus on the compromise that as society we have with children and the future that belongs to them. The discussion turns around the COVID-19 pandemic in the context of children and teenagers, and the need to change world paradigms to preserve the planet and its diversity for them, emphasizing education and psicology as fundamental themes for children development. A claim for training in resilence is included.
\end{abstract}

key words: sustainability goals, pandemics, children future, actions to be taken

\section{Introducción}

Desde hace días me concentro en un artículo que refleje lo que siento en este comienzo de septiembre. Los niños y la COVID-19, fundamental de cara al futuro.

Hace unos meses, Ponte-Sucre (2020) escribía: Las decisiones que pueblos y gobiernos hagamos en las próximas semanas cambiarán la faz del mundo donde vivimos. Salud, educación, economía, cultura, etc. Debemos buscar soluciones a corto plazo para paliar la crisis, y simultáneamente diseñar el mundo en el cual queremos seguir viviendo, la herencia de los niños de hoy, quienes son los principales protagonistas del mundo del futuro. Esta crisis nos da una oportunidad de oro, en el marco de los denominados Objetivos de Sustentabilidad (OdS) (Sacks, 2012), de replantearnos cómo, como humanidad, queremos seguir.

Convencida de esto y pensando en los niños que, en este mes de septiembre -en el hemisferio norte-, deben regresar a las escuelas, cavilo frecuentemente en lo complejo del tema. Nutrición, salud global, interacción social,

\footnotetext{
1 Profesor Titular Emérito, Cátedra de Fisiología, Escuela Luis Razetti, Facultad de Medicina, Universidad Central de Venezuela y Miembro Correspondiente, Academia de Ciencias Físicas, Matemáticas y Naturales de Venezuela. aiponte@gmail.com
} 
violencia, miedo. El reflejo de un mundo desencajado para todos, y que en el caso de los niños va acompañado de la natural incomprensión de los eventos que ocurren a su alrededor.

Keles (2020) afirma que la infección por SARS-CoV-2 en niños es suave. Invoca diferencias en la respuesta inflamatoria e inmune, la secreción de melatonina, el perfil de linfocitos, los niveles de ACE2, una menor exposición a contaminantes ambientales e inmunidad cruzada con otros virus, como razones para esta diferencia. Es cierto además, que usualmente los niños sufren enfermedades más suaves y con menor mortalidad. En el caso de la COVID-19 no existe una explicación adecuada para esta diferencia en la severidad de la enfermedad en los distintos grupos etarios, y se invoca que factores como la comorbilidad y la edad pudieran ser determinantes en la severidad de la infección.

Pero también es cierto que esta pandemia se ha llevado a demasiadas personas, y ha afectado a demasiadas familias, comunidades y grupos sociales a nivel mundial, más aún en países como nuestra herida Venezuela con un perfil de crisis humanitaria compleja indescriptible. La vida diaria de demasiadas personas ha cambiado radicalmente, las economías han caído en recesión y muchas de las tradicionales redes culturales, económicas y públicas de la sociedad se encuentran en situación de tensión sin precedente.

Los niños no son la cara frontal de la crisis, pero pudieran ser víctimas encubiertas debido a los efectos indirectos de la pandemia sobre costumbres de higiene y educación, impacto socioeconómico familiar. Los niños de todos los países están afectados por la situación, y a veces las medidas implementadas para mitigar el contexto podrían causar más daño que beneficio.

Las Naciones Unidas (ONU, 2020) y la Comisión Económica para América Latina y el Caribe (CEPAL, 2020) han confirmado que la COVID-19 ha elevado a los OdS a unos límites prácticamente inalcanzables. Terribles noticias que sugieren que la mayoría de los OdS para minimizar pobreza, proteger el ambiente y alcanzar el bienestar a nivel mundial para 2030 están fuera de alcance actualmente. Es tan dramática la situación que la ONU y sus asociados discuten si los OdS son los adecuados para la época postpandemia.

Por ejemplo, minimizar las muertes prevenibles en niños menores de cinco años y asegurar la asistencia de los niños a la escuela eran objetivos que habían avanzado parcialmente. Al día de hoy, el reloj del desarrollo se ha detenido. Inclusive, estrategias sofisticadas como vacunaciones masivas están estancadas en muchos países. El cierre de las escuelas ha mantenido a $\approx 90 \%$ de los estudiantes a nivel mundial fuera de la escolaridad formal. Según la ONU (2020) este número alcanza 1200 millones de jóvenes y niños en todos los niveles de enseñanza, y la CEPAL (2020) afirma que de ellos, 160 millones se encuentran en América Latina y el Caribe. Adicionalmente como lo resalta la Organización Mundial de la Salud OMS (2020), muchos países no podrán registrar lo que ocurre ya que la recolección de datos persona a persona está obstaculizada por las cuarentenas.

\section{2. ¿Qué hacer entonces?}

Una noticia aparecida en la revista Nature (2020) describe que aún antes de la pandemia un grupo de asesores de la ONU sugería que para asegurar el cumplimiento de los OdS era necesario redistribuir los objetivos en áreas o puntos focales, seis para ser precisos: bienestar (incluyendo eliminación de la pobreza, mejora de la educación y la salud); economías sustentables; acceso a comida y nutrición saludable; acceso a fuentes de energía adecuadas; desarrollo sustentable; y situaciones ambientales (combinando biodiversidad y cambio climático). Alternativamente, otro grupo (La red de soluciones para los Objetivos de Desarrollo Sustentables (SDSN por sus siglas en inglés) sugiere que otra forma de distribuir los 17 OdS sería: educación; género e inequidad; salud (incluyendo demografía y bienestar); descarbonización e industria sustentable; comida; tierras aguas y océanos; ciudades sustentables y comunidades; y revolución digital para el desarrollo sustentable. Sin embargo, Guido Schmidt-Traub, director ejecutivo the SDSN insistió a la revista Nature en que los OdS deberían 
ser alcanzados y se debería desacoplar el crecimiento económico del logro de los OdS. Pero, ¿cómo alcanzar los OdS sin crecimiento mundial?

El mundo está invadido por el SARS-CoV-2 e inmerso en una recesión sin precedentes; los gobiernos están cooperando definitivamente menos, los acuerdos y reuniones relativos a proteger el clima están siendo postpuestos, y la ayuda a los países más desasistidos está cada vez más comprometida. Es fundamental entonces adecuar los OdS a la situación de la pandemia, que está alterando radicalmente las realidades sociales y económicas.

\section{Pero volvamos a los protagonistas de esta reflexión, los niños}

Los padres saben que los niños son eficientes en capturar y transmitir infecciones respiratorias. La investigación científica refrenda esta intuición de los progenitores. Los niños son potenciales elementos efectivos en la distribución y transmisión del SARS-CoV-2 a la población general. Este hecho, aunado a los hábitos sociales y de comportamiento de los niños pequeños y sus compañeros en las escuelas y los hogares de cuidado diario, ha prendido, como refiere Edmunds (2020), las alarmas, debido al riesgo de amplificación de la transmisión del virus en estos contextos y en la población general.

Por ello, al comenzar esta devastadora pandemia, la decisión natural fue cerrar las escuelas y los hogares de cuidado diario y así intentar prevenir la transmisión, a pesar de que, como menciona Heald Sargent y colaboradores (2020), "no existen suficientes evidencias para asegurar que los niños contribuyan de forma importante como fuente de transmisión comunitaria en la distribución del virus".

\subsection{Estar fuera de la escuela}

La UNESCO (2020) ha estimado que más de $60 \%$ de los estudiantes a escala mundial ha tenido una disrupción en sus cursos académicos debido al cierre de las escuelas durante la pandemia de COVID-19. Esto sugiere que con más de 1200 millones de niños y jóvenes fuera de la escuela a nivel mundial, son necesarias medidas de nuevo cuño, posiblemente basadas en tecnologías de la información y comunicación para seguir el proceso de enseñanza-aprendizaje. La revolución tecnológica reciente fundamenta este aprendizaje virtual. Sin embargo, como insiste Edmunds (2020), "hay que darle una dimensión humana, para minimizar las potenciales consecuencias que el aprendizaje virtual podría tener en los procesos sociales, psicológicos y educativos de niños y jóvenes".

Pero, ¿cómo está cada país abordando la situación de su población local, con y sin internet? ¿Hasta dónde llega la situación de inequidad que significa que cada niño con acceso adecuado a internet pueda continuar su aprendizaje, mientras que cada niño sin acceso a internet no podrá continuar su proceso educativo? La respuesta a estas complejas preguntas puede ayudarnos a comprender la realidad donde estamos inmersos. Es fundamental ser objetivos en cuanto a cuál es la capacidad de la sociedad de mantener la escolaridad virtual a pesar de las inequidades de cada grupo en cada país y las inequidades entre los países, debido al reto de implementar los sistemas tecnológicos en cada contexto. Esta premisa debe además tomar en consideración los ingresos reales de las familias, los estados y los países; tener internet con o sin ancho de banda/con o sin el hardware apropiado. En términos francos, y tal y como lo menciona la UNESCO (2020), "la situación actual significa una reversión importante en el indicador de escolaridad, con incalculables consecuencias en el desarrollo humano".

Estar fuera de la escuela por tanto tiempo puede impactar a largo plazo el aprendizaje, y la forma de ser de cada una de esas 1200 millones de almas, los niños y jóvenes. ¿Qué pasará entonces en este 2020 a nivel global con esta situación? En Venezuela llevamos oficialmente cuatro meses (de mediados de marzo a mediados de julio) sin escolaridad presencial; siendo conservadores, esto significa el 50 \% del tiempo del año escolar 2019-2020 
fuera de los espacios de las escuelas. El nuevo año escolar ha comenzado en septiembre, rodeado de incertidumbre. Si a esto sumamos lo precario de nuestra conectividad a internet, podemos pensar que la tasa de escolaridad de cualquier tipo, presencial o virtual, es y será muy escasa, imposible saber con certeza cuánto. Sin embargo, es necesario reconocer que la sociedad no se ha quedado de brazos cruzados. Muchas iniciativas han surgido en el país para intentar solventar, al menos parcialmente esta situación. El cuadro 1 refiere a modo de ejemplo, algunas de estas iniciativas implementadas en tiempos de pandemia.

\section{Cuadro 1}

Algunas iniciativas en Venezuela, labor educativa en pandemia y la revolución tecnológica

\#Alianza Sadpro-UCV, Consejo Científico Tecnológico y Secretaría de Educación de Miranda implementó un plan de actividades de formación en contingencia de Miranda. https://cutt.ly/RfPrTZN

\# Avoquim ha ofrecido experiencias de actualización de docentes en química con enfoque lúdico y su impacto en el desarrollo de la resiliencia ante la COVID-19. \#LatinXChem, \#LatinXChemED \#ED55 @avoquim.

https://avoquim.com

Facilitadora: Amalia Torrealba, química y educadora, directora de AVOQUIM.

\# Banco del Libro ha ofrecido talleres y cursos vacacionales bajo la modalidad virtual, principalmente mediante el uso del Foro-chat de WhatsApp y Google Classroom. @bancodellibro, https://www.facebook.com/bdellibro/

Taller: Ahora que vamos despacio vamos a contar CRISTALES: Taller. Experiencia de indagación que, a través de demostraciones, libros informativos, juegos y cuentos invitó a descubrir juntos secretos de algunos cristales imprescindibles para la vida: La sal y el azúcar.

Facilitadoras: Amalia Torrealba, química y educadora, directora de AVOQUIM y Olga T. González Yunis, bióloga y promotora de lectura del Banco del Libro.

Cursos: Los cuentos de hadas siguen entre nosotros: En los libros, en el cine y la TV. Para mayores de 12 años y Las tres niñas en el Espejo: La materia oscura como novela de aprendizaje (femenino). Para docentes, adultos que trabajan con jóvenes y profesionales.

Facilitadora: María Fernanda Rincón, Banco del Libro.

\# Evelyn Benzecry profesora de artes plásticas, creó su canal de youtube para sus alumnos. https://www.youtube.com/channel/UCnh6QTvnERKWccywn49b1A

\# La Academia Sol de ballet, en Lechería, Estado Anzoátegui ha continuado sus actividades en pandemia. Su estrategia, clases presenciales a pequeños grupos, clases en línea vía WhatsApp, Zoom e Instagram.

https://Academiasol.com.ve

\# Vitrina creativa muestra la primera exposición virtual de recursos didácticos elaborados en cuarentena en estos links. Youtube: https://www/youtube.com/channel/UC7yplbYAZvyCDhBN6FjZT5Q/videos Twitter:

https://twitter.com/ExposiciónP Facebook: https://www.facebook.com/Exposición-PFC-103032511516261

\subsection{Distanciamiento físico y la enseñanza virtual}

A medida que septiembre avanza y se acerca el momento de comenzar el año escolar, aumenta la preocupación por el impacto negativo que el distanciamiento físico y la enseñanza virtual podrían tener en los niños y en el desarrollo de sus habilidades sociales. Una consecuencia de ello para estas generaciones actuales de niños es que podrían transformarse en adultos con escasas capacidades de interacción humana.

Tal y como describe Edmund (2020), las habilidades sociales son como los músculos, si no se usan se atrofian; y esto no es un proceso mental, es un proceso biológico. Una de las principales funciones es ejercitar nuestra fortaleza emocional frente a las adversidades. De hecho, incluso los más solitarios de nosotros necesitamos de compañía en algún momento y las personas que no interactuan socialmente pueden tener ataques de ansiedad 
con mayor frecuencia. Igual como los animales, los humanos solitarios son menos capaces de defenderse de los ataques externos.

Y Edmund (2020) detalla cómo el estar solo es tan adverso fisiológicamente como tener hambre o sed. Al igual que no comer al tener hambre o no beber al tener sed, al no fomentar las relaciones sociales, al estar solo, se producen cambios negativos cognitivos emocionales y fisiológicos. Una máscara de tristeza, irritabilidad, rabia o letargia son reacciones fisiológicas frente a una situación en la cual los lazos afectivos han sido cortados de raíz. Las interacciones espontáneas con extraños, la camaradería con amigos, los compañeros de escuela, las clases de cualquier cosa son oportunidades de encuentro y de alimento social y emocional que constituyen parte de nuestra fisiología.

Queda claro que estos encuentros diarios con otras personas nos reafirman que pertenecemos a un grupo humano, a una red de relaciones interpersonales que alimentan la conciencia y el sentido de pertenencia. Lo contrario, el aislamiento social destruye esa red. La privación de esa relación nos transforma en seres cuyos pensamientos están orientados a la supervivencia. Perdemos la facultad de responder adecuadamente a las señales que nos envía el mundo externo; nos transformamos en individuos completamente neurovegetativos, preparados para pelear o para huir frente a cualquier situación. Los adolescentes especialmente pueden sufrir de esta deprivación social, por ello la interacción con sus pares es esencial para su desarrollo.

De hecho, cada interacción en sociedad, por mínima que sea, implica innumerables juicios intuitivos, interpretar palabras, gestos y expresiones y reaccionar apropiadamente. Como reitera Edmund (2020), "hemos de intuir cuánto compartir, con quién, en qué momento y con cuánta rapidez". Normalmente nuestra práctica en estos temas es continua y por lo tanto nuestro comportamiento es casi como respirar o tragar, no se piensa. Pero la disminución de las oportunidades y las relaciones sólo virtuales y poco espontáneas hacen que se pierda el entrenamiento.

\subsection{Actividad física}

Guan, H., Okely, A.D., Aguilar-Farias, N., Borja del Pozo, C., Draper, C.E., El Hamdouchi, A., Florindo, A.A., Jáuregui, A., Katzmarzyk, P.T., Kontsevaya, A., Löf, M., Park, W., Reilly, J.J., Sharma, D., Tremblay, M.S, Sanne, L.C., Veldman, S.L.C. (2020) describen de forma muy clara cómo los niños son seres inquietos que necesitan actividad física y cómo las guias internacionales recomiendan que los niños en edad preescolar (3-4 años) acumulen al menos 180 minutos diarios de actividad física y no estén sentados frente a una pantalla más de una hora por día, y tengan de 10 a 13 horas de sueño reparador. Para los niños entre 5-17 años estas guias recomiendan tener unos 60 minutos de actividad física vigorosa, no más de dos horas de actividad recreacional sedentaria y entre 9 y 11 horas de sueño diarios.

Es obvio que la forma usual como los niños obtienen su actividad física diaria proviene principalmente de su contacto diario en la escuela, en los parques y las plazas, con recreos, deportes organizados, juegos y danza y que su tiempo sedentario y de sueño transcurre en el hogar. Pero la COVID-19 ha alterado dramáticamente las rutinas de actividad/sedentarismo de los niños y, por ende, sus oportunidades de alcanzar las metas adecuadas de movimiento diario. Esto es especialmente significativo debido a los cierres de las escuelas y el denominado distanciamiento físico implementado en muchos países. De hecho, Guan y colaboradores (2020) aseguran que, en las actuales circunstancias, los niños son menos activos y más sedentarios, con patrones de sueño consistentemente alterados, y con días escolares no estructurados. Incluso reiteran que ya desde antes de la llegada de la pandemia, a nivel mundial, sólo $1 / 5$ de los preescolares y un $10 \%$ de los niños en edad escolar cumplían en promedio las recomendaciones de actividad establecidas. 


\subsection{Confinamiento}

Es de esperar que la situación de pandemia comprometa aún más esta y otras facetas de la salud infantil. Por ejemplo, detallan Guan y colaboradores (2020) cómo el confinamiento en casa, en pequeños espacios y en ambientes internos podría traducirse en deficiencias de vitamina $D$, o alteraciones en la salud mental, e incluso en aumentos de los casos de niños con miopía. Y prosiguen, refiriendo cómo la actividad física prepara a los niños para ser menos propensos a infecciones respiratorias. Esto no toma en cuenta las ventajas indirectas adicionales- que derivan de tener actividad física regular como uno de los mecanismos para aumentar la resiliencia necesaria para enfrentar las situaciones de stress de la vida diaria. En el cuadro 2 se incluyen algunas medidas sencillas que pueden servir de apoyo para fomentar la resiliencia en niños, y también en adultos. En conclusión puede aseverarse que la falta de actividad podría traducirse a la larga en que los niños sean menos activos en general, sean más adictos a las pantallas y duerman peor. Aunado a esto, hay que recordar que si adicionalmente el niño vive en el seno de una familia con vulnerabilidad familiar, donde el distanciamiento físico es imposible, y para quien la actividad física ocurre solo cuando va y viene de la escuela -espacio que muchas veces constituye su único lugar seguro- la situación puede hacerse realmente dramática.

Como se ha mencionado, a medida que la pandemia se expande, las cuarentenas para disminuir la probabilidad de transmisión se han transformado en un escenario lugar-común. En este contexto, muchos gobiernos deben tomar decisiones muy difíciles acerca de cómo mejor proteger a sus pobladores, por ejemplo, sus niñas y mujeres. De hecho, tal y como lo describe Cousins (2020) hay muchas mujeres y niñas en situación de desesperación; las cifras alcanzan valores muy altos y las situaciones podrían llegar a ser devastadoras y con consecuencias impredecibles en la salud de las niñas y mujeres, la de sus familias y la de las comunidades donde habitan. Cousins (2020) nos habla de unos 7 millones de embarazos no deseados que podrían ocurrir debido a esta crisis mundial, con miles de muertes potenciales por abortos no seguros y nacimientos complicados debido al acceso inadecuado a los servicios de emergencia, amén de la situación de violencia doméstica, una pandemia dentro de la pandemia. De igual forma predice que la ausencia de los servicios de salud podría resultar en unos 9,5 millones de mujeres y niñas vulnerables al perder acceso a procesos de contracepción y aborto seguros en 2020. La lamentable consecuencia es que es evidente que las inequidades puestas de relieve por la pandemia se están profundizando, lo que hace imprescindible implementar acciones contundentes y efectivas para solventar estas situaciones.

\subsection{Nutrición}

Adicional a estas circunstancias es preocupante el número de niños que entrarán en malnutrición y desnutrición avanzada como consecuencia de esta crisis global, social y económica sin precedentes, causada por la pandemia de COVID-19. La pérdida de dinero de los hogares, su incapacidad de adquirir alimentos y la interrupción de las cadenas de salud y los servicios de protección serán nefastos para la población infantil. En general, una en diez muertes de niños menores de 5 años es atribuible a una desnutrición severa; esto debido a que los niños están en mayor riesgo de mortalidad por enfermedades infecciosas si están desnutridos. Antes de la COVID-19, UNICEF (2020) estimaba que unos 47 millones de niños a nivel mundial estaban en situación moderada a severa de desnutrición, especialmente en el África subsahariana y en Asia. Venezuela no escapa a este drama en el contexto de la crisis humanitaria compleja que vivimos, ampliamente refrendado por la encuesta ENCOVI (2019). 


\section{Cuadro 2}

Mecanismos que contribuyen a fomentar la resiliencia familiar

\section{Para estimular la resiliencia en el entorno familiar promueve eventos, condiciones y características en niños y familias, que aumenten su capacidad de adaptación a la adversidad y los chances de un desarrollo positivo con base en una interacción positiva entre el niño y su entorno}

\#1 Mantén conexión cariñosa y atenta con tus hijos. Utiliza el tiempo juntos; juega, lee, pasea, conversa, incluso si debe ser por teléfono o dispositivos electrónicos.

\#2 Cubre dentro de tus posibilidades las necesidades básicas de tus hijos. Pide ayuda en caso de urgencia, esto es una fortaleza, no una debilidad. Acude a los lugares donde sientas seguridad y puedas pedir ayuda.

\#3 Permanece atento a la tristeza, ansiedad o rabia que puedan presentar tus hijos. Conversa con ellos, asegúrales con palabras y acciones tu amor.

\#4 Escucha y atiende a tus hijos en momentos de sobresalto. Reafirma con ellos episodios positivos y de esperanza. Apóyate en los maestros en momentos duros.

\#5 Atiende tus propias necesidades. No te sobrecargues de trabajo y responsabilidades. Descansa. Invertir energía en cubrir tus demandas capacita mejor para ayudar a tus hijos y sobrellevar tiempos duros.

\#6 Mantente atento a que tanto tú como tus hijos conserven conexión social con familiares y amigos a través de llamadas, correo, deportes, actividades culturales, educativas y de esparcimiento.

Modificado de Dym y Vivrette, 2020

Las disrupciones de los sistemas económicos, de salud y alimentarios resultantes de la pandemia contribuirán a exacerbar estos niveles de malnutrición. La ONU (2020) estima que unos 140 millones adicionales de personas comenzarán a vivir en pobreza extrema con menos de 1,90 \$ por día en 2020. Y de acuerdo al programa mundial de alimentación el número de personas en los países más desasistidos y enfrentando inseguridad nutricional podría llegar a unos 265 millones de personas al final del 2020. En términos prácticos, según describe Headey y colaboradores (2020), esto representa unos 6-7 millones de niños con desnutrición severa en 2020 y unas 128 605 de muertes adicionales (con un intervalo entre 111193 y 178510 para el mejor y el peor escenario) de niños menores de 5 años que ocurrirán en estos contextos, comparado a si no existiera la pandemia de COVID-19. La conclusión es tremenda, la dignidad de las familias, comunidades y países se encuentra entonces terriblemente expuesta.

\subsection{Emociones}

Finalmente, y en medio del devastador número de muertes y de personas hospitalizadas en esta pandemia, es obligatorio reseñar los efectos psicológicos de la misma en los niños. Por ejemplo, la cobertura periodística sobre los eventos asociados a la salud pública actual afecta emocional y físicamente a nuestras comunidades. Estas noticias son atentamente observadas por nuestros niños. Este hecho hace imprescindible tener a mano explicaciones apropiadas para su edad, a fin de asegurarnos de que nuestros niños tengan una narrativa adecuada que le de soporte a sus experiencias emocionales en este contexto. Esta necesidad es máxima si una persona cercana está hospitalizada o muere por COVID-19.

La COVID-19 afecta predominantemente a los adultos. Los pacientes son deliberadamente aislados de amigos y familia. Como resultado de esto los niños -para quienes el paciente constituye una relación emocional importante- son poco visibles para los familiares adultos y el personal de salud que está a cargo del paciente. Es labor de los cuidadores, del personal de salud y de las familias identificar si los niños están afectados por lo que 
observan, y promover y facilitar una comunicación efectiva con cada niño para quien el paciente hospitalizado es alguien importante. La calidad de comunicación en estas circunstancias es prioritaria. La COVID-19 es una enfermedad con alto riesgo de muerte, concepto que sabemos tiene un efecto psicológico importante en los niños.

De hecho, Rapa, E., Dalton, L., Stein, A. (2020) explican cómo los niños menores de 3 años no son capaces de entender a cabalidad el significado de muerte, pero son susceptibles al efecto indirecto de enfermedades serias y de fallecimientos en la familia. De hecho, los niños son observadores astutos de su ambiente. Ya desde el primer año de vida son capaces de internalizar cambios en el comportamiento y el humor de quienes los cuidan y conforman su entorno más cercano, y niños menores de dos años se sienten angustiados cuando sus cuidadores los dejan para estar pendientes de cuándo regresan. A partir de los 3-4 años los niños entienden el concepto de muerte como una "ida" sin comprender mucho el concepto de irreversibilidad de la muerte por lo cual es importante repetir el mensaje clave que menciona Rapa (2020) "que la persona fallecida no regresará". Solo cuando cumplen 5-6 años los niños reconocen la irreversibilidad y la finalidad de la muerte y su mortalidad personal, pero es a los 10 años cuando los niños son capaces de adquirir la comprensión total de la muerte.

Otro aspecto importante de mencionar es cómo ven y comprenden los niños la causalidad de la enfermedad y la transmisión. Esto se desarrolla con la edad, al comienzo existe un pensamiento mágico, que Rapa y colaboradores (2020) describen como "que los pensamientos pueden causar eventos externos". Es decir, el desarrollo del sentido de conciencia y responsabilidad ocurre, pero que hay que estar atentos a cómo se desarrolla en los niños, lo cual es trascendental ya que lógicamente y debido a sus edades, hay una comprensión muy básica y simple de cómo se transmite la enfermedad. Por ejemplo podría ocurrir que los niños fácilmente atribuyan la causa de la enfermedad a ellos mismos, como menciona Rapa y colaboradores (2020), "como un castigo por su comportamiento equivocado". Incluso, aun durante la adolescencia, una insuficiente o distorsionada información acerca de un evento inesperado y súbito -en cuanto a la muerte- puede resultar en sentimientos de culpabilidad en los jóvenes "por no haber prevenido la causa de muerte de sus padres".

Esos sentimientos de responsabilidad pueden exacerbarse en el contexto de un problema de salud pública como el que vivimos. Por ello, la comunicación debe ser concreta y específica para evitar inferencias incorrectas y malas interpretaciones acerca de cómo alguien cayó enfermo o murió. Los eufemismos, usados para bajar el tono a las noticias compartidas de una muerte, pueden a veces ser confusos y crear más caos. Por ejemplo, cuando se dice "perdimos al abuelo", en el imaginario del niño esto puede significar que lo encontraremos de nuevo.

\subsection{Adultos y padres}

Instintivamente los adultos queremos proteger a los niños de la angustia, especialmente cuando nosotros mismos estamos preocupados y desconcertados. La incertidumbre acerca de cómo y qué compartir con los niños puede ser complicada en el contexto de la trayectoria no predecible de la enfermedad y de los enfermos. Sin embargo, incluso los niños pequeños perciben cambios sutiles en el comportamiento de los adultos cuando alguien enferma o se siente mal (por ejemplo, ver o escuchar personas llorando y conversaciones en voz baja). Si no hay explicaciones los niños sacarán sus conclusiones acerca de lo que ocurre y enfrentarán estas situaciones sin ayuda.

Los padres requieren apoyo para hablar con los niños en estas ocasiones de enfermedad y muerte. Esta necesidad aumenta cuando el aislamiento es intenso y los padres no tienen ni el espacio ni el tiempo para preparase en cómo compartir. Por ello, aunque el personal de salud enfrenta situaciones críticas sin precedentes y presiones emocionales exacerbadas, entre otras causas, por la ausencia de las consultas cara a cara, fundamentales para desarrollar relaciones con los familiares, esos especialistas deben jugar un rol activo en cómo ayudar a las familias a enfrentar estas conversaciones casi imposibles. 
Rapa y colaboradores (2020) insisten en que la COVID-19 representa una infinidad de retos sin precedentes para el cuidado de las personas, los servicios públicos y las comunidades a lo largo y ancho del mundo. Empoderar a los adultos para comunicarse con los niños acerca de la enfermedad y la muerte tiene el potencial de mitigar los efectos psicológicos a corto y largo plazo. Las noticias que los niños enfrentan actualmente son casi imposibles de verbalizar por ellos. Es esencial encontrar las palabras y la forma de darle voz a su experiencia y prevenir que millones de niños a nivel mundial se enfrenten a miedos e incertidumbre.

\section{Conclusiones}

Todo lo discutido resalta la imperiosa necesidad de colocar a los niños en el centro de nuestra atención en esta situación de pandemia por la COVID-19. Esto significa un cambio radical. De hecho, el Comité de Derechos de los Niños de la ONU (2020) declara en grave riesgo los derechos de los niños en esta situación de pandemia. Una argumentación irrefutable de este comité es que "lo que es bueno para los niños es bueno para los países, y las inversiones realizadas en la salud de los niños provee beneficio para las sociedades y los países en el medio y largo término y entre generaciones". Hasta ahora las respuestas de los países a la situación de pandemia se han enfocado en medidas a corto plazo para la protección social y económica y no de largo alcance para la recuperación y la creación de una sociedad equitativa y sana. Como enfatiza la comisión conjunta de la OMSUNICEF-The Lancet (2020), es un deber de los líderes mundiales colocar la salud de los niños en el centro de los planes de recuperación después de la COVID-19 e incluir expertos en asuntos de los niños en las fuerzas legislativas y los grupos de trabajo, a fin de ocuparse juntos por el bienestar de los niños y adolescentes, e incluso, preguntarles a ellos qué necesitan que cambie.

La pandemia es una oportunidad de oro para entender la necesidad de implementar procesos multisectoriales de protección y ejecutar agendas centradas en el niño. El mensaje es claro: no podemos volver a la economía "normal" como la entendimos hasta 2019. Tomar acciones a favor de los niños significa tomar acciones a favor de la protección del ambiente y lograr cambios contundentes en las políticas mundiales para disminuir la emisión de gases de carbono, así como el costo humano asociado a los múltiples desastres ambientales que vemos constantemente. Un mundo centrado en los niños debería ser un mundo más sano, más limpio y más proclive hacia los individuos y las sociedades, y no hacia lo económico y las ganancias como principal motivador.

Los líderes, los expertos, los viejos, los ciudadanos comunes, todos, en un acto de humildad debemos trabajar para lograr que los pobladores de esta nuestra tierra tengamos en todos los países una vida mejor, ahora, y de cara al futuro. Moldear la realidad de hoy para lograr un mundo sustentable para los niños. Asegurar a las familias, las comunidades, los países, la dignidad, los recursos y el tiempo para crecer con sus hijos, en un planeta sano.

Finalizo con una conmovedora frase de mi amiga Annarella Poggioli (2020): Observaba a una mamá con su bebé. Ella le hacía carantoñas, él se reía y participaba en el amoroso juego. Su madre era lo máximo, lo más importante de su mundo, sin máscara o con ella. Siempre será así; lo más importante, el vínculo amoroso, compasivo, de ayuda, de entrega, prevalece y nos queda, además de la ciencia, para sobrevivir cualquier crisis. La historia lo ha verificado una y otra vez.

\section{Agradecimiento}

A las Profesoras Mercedes Schnell y Aura Marina Boadas por la lectura crítica de este artículo y sus acertados comentarios. 


\section{Referencias bibliográficas}

CEPAL-UNESCO. Informe. (2020) La educación en tiempos de la pandemia de COVID-19. Agosto 2020.

Recuperado de: https://cepal.org/es/publicaciones/45904-la-educacion-en-tiempos-de-pandemia-covid19

Cousins, S. (2020). Reporting on COVID-19 has "devastating" effect on women and girls. From the Human Rights Watch report on access to contraception in Brazil.

https://www.hrw.org/news/2020/06/12/brazilprotect-sexual-reproductive-rights-pandemic. The Lancet, 396: 301-302.

Dym Bartlett, J., Vivrette, R. (2020). Ways to Promote Children's Resilience to the COVID-19 Pandemic. Child Trends. Abril 2020. Recuperado de: https://www.childtrends.org/publications/ways-to-promote-childrensresilience-to-the-covid-19-pandemic

Edmunds, W.J. (2020). Finding a path to reopen schools during the COVID-19 pandemic . The Lancet. Agosto 03. Recuperado de: https://doi.org/10.1016/S2352-4642(20)30249-2

Encuesta de Calidad de Vida (Encovi) 2019/20. (2020) Informe de resultados. Recuperado de: https://www.proyectoencovi.com/informe-interactivo-2019

Guan, H., Okely, A.D., Aguilar-Farias, N., Borja del Pozo, C., Draper, C.E., El Hamdouchi, A., Florindo, A.A., Jáuregui, A., Katzmarzyk, P.T., Kontsevaya, A., Löf, M., Park, W., Reilly, J.J., Sharma, D., Tremblay, M.S, Sanne, L.C., Veldman, S.L.C. (2020) Promoting healthy movement behaviours among children during the COVID-19 pandemic. Lancet Child Adoles Health. April 29. Recuperado de: https://doi.org/10.1016/S23524642(20)30131-0

Heald-Sargent, T., Muller, W.J., Zheng, X., Rippe, J., Patel, A.B., Kociolek, L.K. (2020). Age-Related Differences in Nasopharyngeal Severe Acute Respiratory Syndrome Coronavirus 2. Letter (E1-E2) downloaded from $J$ of Amer Med Assoc. Recuperado de: https://doi:10.1001/jamapediatrics.2020.3651

Headey, D., Heidkamp, R., Osendarp, S., Ruel, M., Scott, N., Black, R., Shekar, M., Bouis, H., Flory, A., Haddad, L., Walker, N. (2020). on behalf of the Standing Together for Nutrition consortium. 2020. Impacts of COVID-19 on childhood malnutrition and nutrition-related mortality. The Lancet July 27. Recuperado de: https://doi.org/10.1016/S0140-6736(20)31647-0. Online/Comment: https://doi.org/10.1016/S01406736(20)31648

Keles, E.S. (2020). Mild SARS-CoV-2 infections in children might be based on evolutionary biology and linked with host reactive oxidative stress and antioxidant capabilities. New Microbe and New Infect, 36: 100723. Recuperado de: https://doi.org/10.1016/j.nmni.2020.100723

News in Nature (2020). Time to revise the Sustainable Development Goals. Recuperado de: Nature 583: 331332.

Poggioli A. (2020). Comunicación personal

Ponte-Sucre, A. (2020). COVID-19, perplejidad, incertidumbre, acción... Objetivos de Sustentabilidad. Revista de Postgrado de la Facultad de Medicina, en prensa.

Rapa, E., Dalton, L., Stein, A. (2020). Talking to children about illness and death of a loved one during the COVID-19 pandemic. The Lancet. June 04. Recuperado de: https://doi.org/10.1016/S2352-4642(20)301747 
Sachs, J.D. (2012). From the millennium development goals to sustainable development goals. Recuperado de: The Lancet. 379:2206.

The United Nations Development Programme. Informe. (2020) COVID-19 and Human Development: Assessing the Crisis, Envisioning the Recovery. Mayo 2020. Recuperado de : http://hdr.undp.org/en/hdp-covid

The WHO-UNICEF-Lancet Commissioners. (2020). After COVID-19, a future for the world's children? The Lancet. July 02. Recuperado de: https://doi.org/10.1016/ S0140-6736(20)31481-1

World Health Organization. 2020. COVID-19: Resources for Care for Young Children Recuperado de: https://www.who.int/maternal_child_adolescent/links/covid-19-mncah-resources-for-care-for-youngchildren

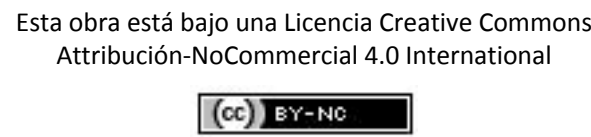

